\title{
Conhecimento da Enfermagem sobre assistência ao paciente disfágico no Hospital Regional do Sudoeste do Paraná
}

\author{
Ana Karenina Cioatto ${ }^{1}$, Nádia Aparecida Zanella²
}

\begin{abstract}
RESUMO
A pesquisa teve por objetivo avaliar o conhecimento da equipe de enfermagem a respeito da disfagia e implicações na assistência ao paciente no ambiente hospitalar. 0 estudo é de campo, descritivo, com aplicação de questionário a 113 profissionais da equipe de enfermagem, sendo 13 enfermeiros, e 100 técnicos de enfermagem, dos diversos setores do Hospital Regional do Sudoeste - PR. A maioria dos profissionais pesquisados evidenciaram apresentar conhecimento satisfatório sobre a disfagia, causas, consequências e identificação adequada dos sinais e sintomas, fatores essenciais para detectar a presença de alteração da deglutição, bem como diagnósticos e cuidados de enfermagem relacionados à disfagia. Contudo, é possível verificar que em alguns itens relacionados à alteração da deglutição, os enfermeiros estão mais preparados que os técnicos de enfermagem. De forma geral, esta pesquisa apontou que há conhecimento teórico e prático a respeito da disfagia e suas relações, favorecendo assistência de maior qualidade ao paciente disfágico.
\end{abstract}

Descritores: Transtornos de Deglutição; Cuidados de Enfermagem; Assistência Hospitalar.

\section{Nursing knowledge about patient care with dysphagia at the Regional Hospital of Southwest of Paraná}

\begin{abstract}
The research aimed to evaluate the knowledge of nursing staff about the dysphagia and implications on patient care in the hospital environment. The study is descriptive, field, with questionnaires to 113 professionals from nursing staff, being 13 and 100 nurses nursing technicians, the various sectors of Southwest Regional Hospital - PR. Most professionals surveyed showed present satisfactory knowledge about dysphagia, its causes, consequences and appropriate identification of the signs and symptoms, essential factors for the presence of a change of swallowing, as well as the diagnoses and nursing care related to dysphagia. However, it is possible to verify that in some items related to change of swallowing, the nurses were more prepared that nursing technicians. In General, this research indicated that there is a theoretical and practical knowledge about the dysphagia and their relationships, favoring a higher quality assistance to the patient disfágico.
\end{abstract}

Descriptors: Swallowing Disorders; Nursing Care; Hospital Assistance.

\footnotetext{
${ }^{1}$ Fonoaudióloga pela Universidade Paranaense (UNIPAR), Umuarama, PR, Brasil.

${ }^{2}$ Especialista em Enfermagem do Trabalho pela Universidade Paranaense (UNIPAR), Umuarama, PR, Brasil.
} 


\section{Introdução}

O processo adequado e eficiente da alimentação faz-se necessário para a manutenção saudável do estado clínico geral do paciente, já que qualquer alteração que dificulte a realização de uma boa deglutição irá repercutir no estado nutricional, respiratório e na qualidade de vida do paciente.

A disfagia, transtorno da deglutição, é um distúrbio que dificulta ou impossibilita a ingestão segura de alimento e/ou saliva, podendo provocar complicações ao quadro clínico do paciente, como desidratação, desnutrição, emagrecimento, alterações respiratórias, como a pneumonia aspirativa e até óbito ${ }^{1,2}$.

A equipe de enfermagem, juntamente com a equipe multidisciplinar que acompanha os pacientes em internamento hospitalar, precisa estar atenta às mudanças significativas do mecanismo da deglutição, seus sinais e sintomas, pois questões pulmonares e nutricionais oriundas da disfagia comprometem ainda mais o quadro clínico desses pacientes, prorrogando o período de internamento, a ocupação dos leitos hospitalares, gerando mais custos para as instituições, além de prolongar a exposição desnecessária do paciente ao ambiente hospitalar.

Percebe-se desde a grade curricular dos cursos de formação de Enfermagem, a ausência de uma abordagem mais completa e detalhada do tema disfagia e suas implicações, sendo um assunto negligenciado portanto, por maior parte das equipes de enfermagem, no dia a dia na assistência hospitalar.

Assim, a provável falta de conhecimento sobre a disfagia, dificulta a sua detecção e intervenção, isto é, a prescrição da Enfermagem de cuidados que favoreçam a uma melhora do estado de saúde geral dos pacientes disfágicos.

Neste sentido, torna-se fundamental desenvolver pesquisas que englobam a disfagia e suas complicações, pois além de consultas, exames e medicamentos, o paciente necessita estar com o estado nutricional equilibrado, facilitando seu processo de recuperação geral, refletindo dessa forma na qualidade de vida.

Esse estudo teve por objetivo avaliar o conhecimento da equipe de enfermagem a respeito da disfagia e implicações na assistência do paciente no âmbito hospitalar. Buscou igualmente, averiguar a acurácia na identificação de sinais e sintomas, além de constatar o papel do enfermeiro na equipe multidisciplinar no atendimento às alterações de deglutição.

\section{Metodologia}

Foi realizada uma pesquisa exploratória, de campo, do tipo descritiva, com abordagem quantitativa, tendo como amostra a equipe de enfermagem. Os sujeitos foram selecionados aleatoriamente, de acordo com a disponibilidade dos profissionais conforme a escala de jornada de trabalho, correspondendo a $50 \%$ de profissionais de enfermeiros e técnicos de enfermagem.

Os setores envolvidos foram: UTI adulta, UTI neonatal, pronto - atendimento, centro- cirúrgico, maternidade, clínica cirúrgica e clínica médica, ambulatório, do Hospital Regional do Sudoeste do Paraná - HRS, o qual compreende 146 leitos, estando atualmente, 102 leitos ativos.

O HRS é um hospital de média - alta complexidade, público, de gestão estadual, tendo sua abrangência 42 municípios localizados no sudoeste do Paraná, atendendo uma população de aproximadamente 650 mil habitantes. As suas principais referências para atendimento são: traumatologia, unidade de terapia intensiva e gestação de alto risco, vinculados as Redes de Urgência e Emergência e ao Programa Mãe Paranaense.

Esta instituição, situa-se na cidade de Francisco Beltrão, na região do sudoeste do Paraná, sendo a cidade pólo regional em saúde. A economia dessa região baseia-se principalmente na agricultura familiar, comércio e pequenas indústrias, permeadas pelas culturas de origem alemã e italiana, predominantemente.

O instrumento principal desse estudo foi um questionário investigativo elaborado a fim de obter informações das variáveis de interesse composto por 20 questões fechadas, em que algumas questões poderiam apresentar mais de uma opção assinalada, que foram aplicadas a 13 enfermeiros e 100 técnicos de enfermagem do Hospital Regional do Sudoeste - Paraná. Dentre as informações destaca-se qual é o conhecimento desses profissionais sobre disfagia, seus sinais e sintomas, intervenções de enfermagem e quais as complicações possíveis para o paciente não diagnosticado e tratado.

Os sujeitos da pesquisa recebiam o questionário durante a realização do plantão, respondendo assim que possível, diante a rotina da prestação contínua dos cuidados, entregando - o na passagem de plantão para a próxima equipe, a qual também receberia o questionário para responder, e a pesquisadora recolhia posteriormente. 
Após coletados, os dados foram dicotomizados, digitalizados em uma planilha Excel (MICROSOFT, 2010) e submetidos à análise estatística descritiva no softaware Statistical Package for the Social Sciences- SPSS 20.0.

O questionário foi aplicado pela própria pesquisadora no mês de agosto e setembro de 2013, nas dependências do Hospital Regional, após a aprovação do projeto de pesquisa pelo Comitê de Ética em Pesquisa via Plataforma Brasil, sob parecer no 341.813 em 26/07/2013.

\section{Resultados}

Verifica-se com os dados coletados, que a maioria dos profissionais encontra-se na faixa etária de 29 - 39 anos, com apenas 6 profissionais acima de 50 anos, prevalecendo o sexo feminino em ambas categorias.

Tabela 1 - Distribuição das características de identificação dos profissionais da equipe de enfermagem do HRS.

\begin{tabular}{|c|c|c|c|c|}
\hline \multirow[t]{2}{*}{ Categorias } & \multicolumn{2}{|c|}{ Técnico de Enfermagem } & \multicolumn{2}{|c|}{ Enfermeiro } \\
\hline & $n$ & $\%$ & $n$ & $\%$ \\
\hline \multicolumn{5}{|l|}{ Gênero } \\
\hline Masculino & 11 & 11,0 & 05 & 38,5 \\
\hline Feminino & 89 & 89,0 & 08 & 61,5 \\
\hline Total & 100 & 100 & 13 & 100 \\
\hline \multicolumn{5}{|l|}{ Faixa Etária } \\
\hline $18-28$ anos & 21 & 21,0 & 02 & 15,4 \\
\hline $29-39$ anos & 50 & 50,0 & 07 & 53,8 \\
\hline $40-49$ anos & 24 & 24,0 & 03 & 23,1 \\
\hline Acima de 50 anos & 05 & 5,0 & 01 & 7,7 \\
\hline Total & 100 & 100 & 13 & 100 \\
\hline \multicolumn{5}{|c|}{ Formação Profissional } \\
\hline Enfermeiro & 0 & 0 & 13 & 100,0 \\
\hline $\begin{array}{l}\text { Técnico de } \\
\text { Enfermagem }\end{array}$ & 100 & 100,0 & 0 & 0 \\
\hline Total & 100 & & 13 & 100 \\
\hline
\end{tabular}

Hospital Regional do Sudoeste do Paraná, 2013

A partir dos dados coletados, observa-se que há uma prevalência de enfermeiros que participaram dessa pesquisa, alocados na Unidade de Terapia Intensiva adulta (30,8\%), e no caso dos técnicos de enfermagem, a maioria encontramse nas clínicas cirúrgicas I e II (25\%). Já sobre o turno de trabalho, a maioria dos técnicos de enfermagem (57\%) trabalha no período diurno, enquanto os enfermeiros trabalham tanto no turno diurno $(46,2 \%)$ quanto no noturno $(46,2 \%)$. Há uma incidência, embora pequena, de técnicos de enfermagem no plantão diurno $(57 \%)$.

Em relação aos setores trabalhados no ambiente hospitalar, tem-se que a categoria profissional de técnico de enfermagem atua ou já atuou mais no setor da clínica médica (23\%) e na clínica cirúrgica (18\%), enquanto com os enfermeiros ocorreu maior prevalência nos setores do pronto socorro, maternidade e clínica cirúrgica (16\%), respectivamente em cada setor.

Quanto ao tempo de formação houve o predomínio de 5 a 10 anos $(76,9 \%)$ para enfermeiros e $(47,0 \%)$ para técnicos de enfermagem. E quanto ao tempo de experiência, tanto para a categoria de enfermeiros $(69,2 \%)$ quanto a dos técnicos de enfermagem $(37,0 \%)$ o intervalo é de 5 a 10 anos.

Analisando os dados coletados, percebe-se que além de haver uma compreensão adequada dos fatores etiológicos da disfagia, a maioria (92\%) dos técnicos de enfermagem considera que a disfagia é um distúrbio da deglutição, caracterizado pela alteração de uma ou mais fases da deglutição, assim como também os enfermeiros em unanimidade.

De acordo com mais de $90 \%$ dos sujeitos pesquisados, o fonoaudiólogo é o profissional responsável pela reabilitação das disfagia.

Com relação ao posicionamento correto do paciente durante a oferta da dieta por via oral, a maioria dos profissionais, $69,2 \%$ dos enfermeiros, e $65 \%$ dos técnicos de enfermagem responderam que seria sentado. 
Tabela 2 - Distribuição dos dados referentes a capacidade de identificar o conceito da disfagia*

\begin{tabular}{c|c|c|c|c}
\hline Categorias & \multicolumn{2}{|c|}{ Técnico de Enfermagem } & \multicolumn{2}{c}{ Enfermeiro } \\
\hline $\begin{array}{c}\text { Distúrbio da fonação, caracterizado pela alteração da } \\
\text { percepção e produção da fala }\end{array}$ & 07 & 7,0 & $\mathrm{n}$ & $\%$ \\
\hline $\begin{array}{c}\text { Distúrbio da deglutição, caracterizado pela alteração } \\
\text { de uma ou mais fases da deglutição }\end{array}$ & 92 & 92,0 & 0 & 0 \\
\hline $\begin{array}{c}\text { Distúrbio da respiração, caracterizada pela sensação } \\
\text { de falta de ar }\end{array}$ & 01 & 1,0 & 13 & 100,0 \\
\hline $\begin{array}{c}\text { Distúrbio do sistema motor, caracterizado por } \\
\text { redução da mobilidade dos membros superiores }\end{array}$ & 0 & 0 & 0 & 0 \\
\hline Total & 100 & 100 & 13 & 100 \\
\hline
\end{tabular}

Hospital Regional do Sudoeste do Paraná, 2013

Tabela 3 - Distribuição dos dados referentes ao posicionamento adequado para o paciente permanecer durante a oferta da dieta oral:

\begin{tabular}{c|c|c|c|c}
\hline Categorias & \multicolumn{2}{|c|}{ Técnico de Enfermagem } & \multicolumn{2}{c}{ Enfermeiro } \\
\hline & $\mathrm{n}$ & $\%$ & $\mathrm{n}$ & $\%$ \\
\hline Deitado & 0 & 0 & 0 & 0 \\
\hline Sentado & 65 & 65,0 & 09 & 69,2 \\
\hline Cabeceira elevada a 45 & 33 & 33,0 & 04 & 30,8 \\
\hline Sentado e Cabeceira elevada a 45 & 02 & 2,0 & 0 & 0 \\
\hline Total & 100 & 100 & 13 & 100 \\
\hline
\end{tabular}

Hospital Regional do Sudoeste do Paraná, 2013

A partir dos dados coletados comprova-se que mais de $90 \%$ dos sujeitos pesquisados reconhecem serem capazes de identificar se o paciente apresenta algum sinal ou sintoma que caracterize uma alteração de deglutição.

Observa-se que $92,3 \%$ dos enfermeiros e $88 \%$ dos técnicos de enfermagem, reconhecem que a tosse ou engasgo ao engolir, redução do estado de alerta e pneumonia aspirativa são sinais importantes na avaliação de uma alteração de deglutição.

Tabela 4 - Distribuição dos dados referentes a itens importantes na avaliação da detecção de alteração da deglutição.

\begin{tabular}{c|c|c|c|c}
\hline Categorias & \multicolumn{2}{|c|}{ Técnico de Enfermagem } & \multicolumn{2}{c}{ Enfermeiro } \\
\hline & $\mathrm{n}$ & $\%$ & $\mathrm{n}$ & $\%$ \\
\hline Tosse ou engasgo ao engolir & 09 & 9,0 & 1 & 7,7 \\
\hline Redução do estado de alerta (consciência) & 01 & 1,0 & 0 & 0 \\
\hline Pneumonia aspirativa & 01 & 1,0 & 0 & 0 \\
\hline Retenção urinária & 88 & 88,0 & 12 & 92,3 \\
\hline Redução do estado de alerta (consciência) e \\
Retenção urinária & 01 & 1,0 & 0 & 0 \\
\hline Total & 100 & 100 & 13 & 100 \\
\hline
\end{tabular}

Hospital Regional do Sudoeste do Paraná, 2013

Os dados analisados demonstram que $61,5 \%$ dos enfermeiros, e $72 \%$ dos técnicos de enfermagem, confirmaram como sinais e sintomas da disfagia, a disfonia, dispnéia, engasgo, tosse e queda de saturação durante a alimentação.

Quanto às consequências da disfagia, houve prevalência significativa da alternativa sobre a aspiração laringotraqueal, pneumonia, emagrecimento, desnutrição e/ou desidratação, alteração psicossocial e do prazer alimentar, tanto por parte dos enfermeiros $(76,9 \%)$, quanto dos técnicos de enfermagem $(79 \%)$.

Segundo, $54 \%$ dos enfermeiros e $71 \%$ dos técnicos de enfermagem, ao se depararem com alguma alteração de deglutição, eles confirmaram que registram no prontuário, comunicam ao médico, comunicam o fonoaudiólogo, e no caso dos técnicos de enfermagem, comunicam ao enfermeiro.

\footnotetext{
* Marchesan IQ, Zorzi JL, Gomes ICl. Tópicos em fonoaudiologia. São Paulo: Lovise;1995 apud³
} 
No caso dos enfermeiros, a maioria $(76,9 \%)$ reconhece que o único diagnóstico que não deveria ser prescrito ao paciente disfágico, é o débito cardíaco diminuído, já que os demais são pertinentes à disfagia.

Tabela 5 - Distribuição dos dados referentes aos diagnósticos de enfermagem, de acordo com a NANDA Internacional, que seriam prescritos para pacientes com alteração de deglutição.

\begin{tabular}{c|c|c|c|c}
\hline Categorias & \multicolumn{2}{|c|}{ Técnico de Enfermagem } & \multicolumn{2}{c}{ Enfermeiro } \\
\hline & $\mathrm{n}$ & $\%$ & $\mathrm{n}$ & $\%$ \\
\hline Padrão ineficaz de alimentação & 16 & 16,0 & 01 & 7,7 \\
\hline Risco de aspiração & 05 & 5,0 & 0 & 0 \\
\hline Débito cardíaco diminuído & 72 & 72,0 & 10 & 84,6 \\
\hline Dentição prejudicada & 07 & 7,0 & 0 & 0 \\
\hline Padrão ineficaz de alimentação e Risco de aspiração & 0 & 0 & 2 & 15 \\
\hline Total & 100 & 100 & 13 & 100 \\
\hline
\end{tabular}

Hospital Regional do Sudoeste do Paraná, 2013

Com os dados dessa pesquisa, analisa-se que todos os enfermeiros pesquisados e $91 \%$ dos técnicos de enfermagem, ao admitir um paciente com fatores de risco para alguma alteração de deglutição, não consideram como procedimentos essenciais, a alternativa que afirma fazer nada, além da rotina normal de admissão do doente.

Analisando os dados coletados, observa-se que $92,3 \%$ dos enfermeiros e $80 \%$ dos técnicos de enfermagem, não consideram como cuidado primordial, oferecer dieta por via alternativa de alimentação ou via oral para o paciente na postura deitado e com a cabeça fletida.

\section{Discussão}

Com o objetivo de avaliar o conhecimento da equipe de enfermagem de um hospital de grande porte da região do sudoeste do Paraná, sobre a disfagia e suas implicações na assistência do paciente disfágico, foi realizada uma caracterização dos profissionais, traçando o perfil destes, segundo as seguintes variáveis: faixa etária, gênero, formação profissional, tempo de formação e experiência profissional, setores que trabalha ou já trabalhou no ambiente hospitalar, a unidade e o turno de trabalho em que atuam nesta instituição.

Este estudo foi realizado através da aplicação de um questionário com 113 profissionais da equipe de enfermagem do Hospital Regional do Sudoeste - HRS, no município de Francisco Beltrão. A coleta de dados ocorreu nos meses de agosto e setembro de 2013. Na sequência, estão expostas a análise e discussão dos dados obtidos com esta pesquisa, com a descrição dos dois grupos analisados: enfermeiros e técnicos de enfermagem, através do método quantitativo.

Em relação ao perfil profissional, a maioria dos profissionais estudados encontram-se na faixa etária de 29 a 39 anos, com apenas 6 profissionais acima de 50 anos, o que demonstra ainda ser uma categoria de profissionais jovens, com 0 crescimento significativo da disponibilidade de cursos de enfermagem, a facilitação ao acesso aos centros de ensino, e o aumento da busca por esta profissão, devido ao aumento da demanda no mercado de trabalho atual.

Os enfermeiros foram os segundos profissionais mais requisitados pelo mercado de trabalho nos últimos três anos atrás apenas dos analistas de tecnologia da informação $(\mathrm{TI})^{4}$.

Ainda, um estudo recente do Instituto de Pesquisa Econômica aplicada (Ipea) mostra que são os enfermeiros os profissionais de saúde mais demandados pelo mercado 4 .

O levantamento realizado pelo Ipea revela que, de 2009 a 2012, dos 304.317 postos de trabalho de nível superior abertos no Brasil, 27.282 vagas, quase $9 \%$ do total, foram para enfermeiros ${ }^{4}$.

Diante a magnitude da predominância do público feminino na classe de enfermagem, é previsto a prevalência do sexo feminino tanto na categoria de enfermeiros (61,5\%), quanto na de técnico de enfermagem (89\%).

A partir dos dados observados, verifica-se que há uma prevalência de enfermeiros, atuantes na Unidade de Terapia Intensiva adulta (30,8\%), e em relação aos técnicos de enfermagem, a grande parte encontra-se nas clínicas cirúrgicas I e II (25\%). 
O Ministério da Saúde, por meio da Agência Nacional de Vigilância Sanitária (ANVISA) publicou a RDC nº 7/2010, que dispõe sobre os requisitos mínimos para funcionamento de Unidades de Terapia Intensiva e dá outras providências. No artigo 13 dessa RDC n 7/2010 consta a exigência da designação de um responsável técnico médico, um enfermeiro coordenador da equipe de enfermagem e um fisioterapeuta coordenador da equipe de fisioterapia, assim como seus respectivos substitutos ${ }^{5}$.

Ainda, a RDC n. 07/2010, determina a necessidade de enfermeiros assistenciais, no mínimo 01 (um) para cada 08 (oito) leitos ou fração, em cada turno, como também, elenca sobre os técnicos de enfermagem, ou seja, no mínimo 01 (um) para cada 02 (dois) leitos em cada turno, além de 1 (um) técnico de enfermagem por UTI para serviços de apoio assistencial em cada turno ${ }^{5}$.

A Unidade de Terapia Intensiva (UTI) caracteriza-se como uma unidade reservada, complexa, dotada de monitorização contínua que admite pacientes potencialmente graves ou com descompensação de ou mais sistemas orgânicos. Dispõe de assistência médica, de enfermagem, de uma equipe multiprofissional, com a intervenção de fisioterapeutas, fonoaudiólogos, nutricionistas, psicólogos, assistentes sociais ${ }^{6}$. Ainda, sabe-se que na UTI adulta, há um índice elevado de pacientes com disfagia, já que o grau de comprometimento da saúde no geral é bastante significativo.

Kunigk e Chehter ${ }^{*}$ relatam que as unidades de tratamento intensivo (UTI) são destinadas ao atendimento de pacientes graves ou de risco, por problemas respiratórios, cardiovasculares, neurológicos e outros, com frequentes procedimentos invasivos, entre eles, a intubação oro-traqueal. Esse procedimento geralmente promove complicações de estruturas, como: língua, palato, soalho da boca, úvula, esôfago, laringe, traqueia, entre outras lesões, que comprometem o processo da deglutição.

A gerência do serviço de enfermagem necessita avaliar continuamente a carga de trabalho de sua equipe, utilizando conhecimentos e instrumentos que lhe permitam realizar um melhor planejamento, alocação, distribuição e controle do quadro de enfermagem de acordo com Fugulin*.

"Os sistemas de classificação de pacientes podem ser definidos como métodos que determinam, monitoram e validam as necessidades de cuidado individualizado do paciente" 9 .

A Resolução № 293/04, do Conselho Federal de Enfermagem (COFEN), estabelece os parâmetros para dimensionar o quantitativo mínimo dos diferentes níveis de formação dos profissionais de enfermagem para a cobertura assistencial nas instituições de saúde, fazendo uso do sistema de classificação de pacientes (SCP) ${ }^{10}$.

O SCP determina o grau de dependência de um paciente em relação à equipe de enfermagem, para assim, estabelecer o tempo despendido no cuidado direto e indireto, além do qualitativo de pessoal, para atender às necessidades biopsicossociais e espirituais do paciente, segundo Gaidzinski".

Desse modo, a classificação de pacientes, de acordo com o grau de dependência da equipe de enfermagem, constitui uma das etapas dos métodos de dimensionamento de pessoal, possibilitando o exercício da profissão com mais qualidade e resolutividade das demandas dos pacientes ${ }^{12}$.

Considerado como instrumento essencial na prática gerencial de enfermagem, o SCP:

[...] proporciona, ainda, informações para o processo de tomada de decisão quanto à alocação de recursos humanos, à monitorização da produtividade e aos custos da assistência de enfermagem, bem como para a organização dos serviços e planejamento da assistência de enfermagem. $O$ conhecimento do perfil assistencial dos pacientes é outro fator que pode subsidiar o planejamento e a implementação de programas assistenciais que melhor atendam às necessidades dessa clientela, auxiliando na distribuição diária e na capacitação dos recursos humanos de enfermagem para o atendimento de cada grupo de pacientes ${ }^{12}$.

Quanto aos técnicos de enfermagem do setor das clínicas cirúrgicas, ocorre uma maior rotatividade de pacientes internados por diversas enfermidades, possibilitando a estes profissionais uma maior interação com inúmeros casos clínicos, de origem diversas (neurológicas, traumas, cardiovasculares, doenças genéticas, oncológicas, etc.), facilitando o reconhecimento de sinais e sintomas que levam ao quadro de alguma alteração de deglutição.

\footnotetext{
* Kunigk MRG, Chehter E. Disfagia orofaríngea em pacientes submetidos à entubação orotraqueal. Rev Soc Bras Fonoaudiol. 2007;12(4):287-91 apud7

* Fugulin FMT. Dimensionamento de pessoal de enfermagem: avaliação do quadro de pessoal das unidades de internação de um hospital de ensino [tese doutorado]. São Paulo: Escola de Enfermagem, Universidade de São Paulo; 2002 apud ${ }^{8}$

* Gaidzinski RR. O dimensionamento do pessoal de enfermagem segundo a percepção de enfermeiras que vivenciam esta prática. [tese]. São Paulo (SP): Escola de Enfermagem/USP; 1994 apud $^{11}$
} 
Observa-se a partir dos dados analisados, que a maioria dos enfermeiros que participaram do estudo trabalham tanto no turno diurno, em jornadas de trabalho de 12/36 horas (07:00 - 19:00), quanto no noturno (19:00 - 07:00). A incidência, embora pequena, de técnicos de enfermagem no plantão diurno (57\%), justifica-se pela concentração dos principais procedimentos neste horário.

Conforme os dados expostos, a categoria profissional de técnico de enfermagem atua ou já atuou mais no setor da clínica médica (23\%) e na clínica cirúrgica (18\%), o que demonstra uma aproximação ainda maior com a assistência ao paciente internado, possibilitando uma interação mais direta, contínua com pacientes suscetíveis a apresentar algum tipo de alteração de deglutição, diante a diversidade dos quadros clínicos. Já no caso dos enfermeiros, houve uma predominância maior nos setores do pronto socorro, maternidade e clínica cirúrgica (16\%), respectivamente em cada setor.

Verifica-se nos dados apresentados anteriormente, que quanto ao tempo de formação prevalece o intervalo de tempo de 5 a 10 anos, sendo $76,9 \%$ para os enfermeiros e $47 \%$ para os técnicos de enfermagem. Com relação ao tempo de experiência, tanto para a categoria de enfermeiros $(69,2 \%)$ quanto a dos técnicos de enfermagem (37\%) o intervalo de 5 a 10 anos prevaleceu.

O Hospital Regional do Sudoeste foi inaugurado em 26 fevereiro de 2010, ou seja, é um estabelecimento de saúde recente, 0 que concerne com os dados obtidos nessa pesquisa da prevalência de profissionais jovens, com relativo tempo de formação e de experiência. Provavelmente, isso se deve ao fato da busca por cursos da área de enfermagem terem crescido nos últimos anos.

Analisando os dados da tabela, observa-se que a maioria (92\%) dos técnicos de enfermagem consideram que a disfagia é um distúrbio da deglutição, caracterizado pela alteração de uma ou mais fases da deglutição, assim como os enfermeiros em unanimidade.

De acordo com American Speech-Language-Hearing Association*, a disfagia é :

[...] um distúrbio da deglutição decorrente de causas neurológicas e/ou estruturais. Pode ser decorrente de traumas de cabeça e pescoço, de acidente vascular encefálico, de doenças neuromusculares degenerativas, de câncer de cabeça e pescoço, de demências e encefalopatias. A disfagia mais frequentemente reflete problemas envolvendo a cavidade oral, faringe, esôfago ou transição esofagogástrica. A disfagia ou dificuldade na deglutição pode resultar na entrada de alimento na via aérea, resultando em tosse, sufocação/asfixia, problemas pulmonares e aspiração. Também, gera déficits nutricionais, desidratação com resultado em perda de peso, pneumonia e morte.

Sobre os resultados a respeito dos fatores etiológicos da alteração da deglutição, verificou-se que há uma compreensão adequada de que a disfagia pode ocorrer devido a presença de doenças degenerativas, tumores cerebrais, câncer de cabeça e pescoço. Contudo, alguns profissionais consideraram apenas uma outra opção e não todas as alternativas, já que na pergunta se questionava a exceção dos fatores causais, que no caso, seria a presença de úlcera venosa de pressão.

A disfagia pode iniciar-se de um modo progressivo ou brutal, pode ser aguda ou crônica, intermitente ou constante. Sua etiologia pode ser de origem neurogênica, como acidente vascular encefálico, mal de Parkinson, mal de Alzheimer, miastenia gravis, distrofia muscular, traumas cranianos e câncer de cabeça e pescoço, tumores cerebrais, esclerose lateral amiotrófica, paralisia cerebral, síndrome de Guillan-Barret e encefalopatias anóxicas, entre outras, assinala Marchesan*.

Em relação as consequências que a disfagia pode causar, prevaleceu tanto por parte dos enfermeiros (76,9\%), quanto dos técnicos de enfermagem (79\%), a compreensão de que a aspiração laringotraqueal, pneumonia, emagrecimento, desnutrição e/ou desidratação, alteração psicossocial e do prazer alimentar, são possíveis consequências do quadro clínico da disfagia. Assim, observa-se uma adequada percepção da alteração da deglutição, o que sugere uma certa experiência clínica junto ao paciente disfágico.

Vale salientar, que na rotina hospitalar dessa instituição não há o serviço de Fonoaudiologia no turno da noite, o que poderia justificar o desconhecimento de alguns profissionais quanto a questionamentos referentes a disfagia, já que não recebem diretamente orientações, não acompanham o processo de avaliação e diagnóstico. Além disso, uma prática observada é que a evolução da equipe multiprofissional nos prontuários dos pacientes ocorre separadamente da equipe de enfermagem, 0 que poderia também, favorecer o distanciamento de alguns profissionais com a realidade do paciente disfágico.

Um dos cuidados específicos de extrema relevância é o desempenhado pelo fonoaudiólogo, que é o profissional habilitado para avaliação e reabilitação da disfagia. Sua atuação não se restringe ao atendimento clínico e sessões especializadas, mas inclui orientação, educação, treinamento do doente com disfagia e de seus cuidadores, para a reorganização de uma deglutição eficiente ${ }^{14}$.

\footnotetext{
${ }^{*}$ American Speech-Language-Hearing Association. Model Medical Review Guidelines for Dysphagia Services [monograph on the Internet] 2004 apud ${ }^{14}$

* Marchesan IQ, Zorzi JL, Gomes IC.I. Tópicos em fonoaudiologia. São Paulo: Lovise;1995 apud³
} 
Quanto a equipe de enfermagem, são usualmente os primeiros profissionais a identificarem os sinais e sintomas de disfagia, além de outras condições associadas, como má-dentição ou próteses dentárias deficientes, presença de desnutrição e desidratação. Sendo também, o profissional responsável pela assistência ao paciente durante a oferta da dieta, seja oral ou via sonda e parenteral, higienização oral, e assim, será o responsável direto em implementar as recomendações fonoaudiológicas e nutricionais durante as refeições ${ }^{1}$.

Contudo, infelizmente, observa-se ainda a compreensão de alguns profissionais quanto a predominância da figura do médico na atenção ao paciente hospitalizado, negligenciando a importância e a particularidade de cada profissional participante da equipe multiprofissional, que só vem a contribuir qualitativamente na recuperação global do paciente.

O fonoaudiólogo realiza a avaliação clínica das condições de deglutição do paciente, prescreve e participa ativamente da avaliação instrumental da deglutição, junto ao otorrinolaringologista no exame da videofluoroscopia. Com o diagnóstico estabelecido, esse profissional determina a possibilidade ou não da dieta via oral segura, sem risco de aspiração. Ainda, planeja e aplica um programa terapêutico (terapias indireta e direta), baseando-se nas dificuldades individuais, como também, orienta o paciente e/ou cuidador sobre procedimentos facilitadores durante a administração da dieta via oral, coordenando o processo progressivo da consistência da dieta ${ }^{1}$.

Com relação ao posicionamento correto do paciente durante a ministração da dieta por via oral, a maioria dos profissionais, sendo $69,2 \%$ dos enfermeiros, e $65 \%$ dos técnicos de enfermagem responderam que seria sentado. Todavia, houve uma significante afirmação da posição da cabeceira elevada a $45^{\circ}$ por parte da equipe de enfermagem, demonstrando haver dúvidas quanto ao posicionamento, e assim, pode comprometer o estado clínico e nutricional do paciente. Entretanto, uma possibilidade foi a suposição da impossibilidade de alguns pacientes com quadros clínicos específicos, que não permitem o posicionamento da cabeceira do leito a $90^{\circ}$, ou seja, sentado, desde o impedimento devido a um acesso venoso ou a própria debilidade física do paciente.

Em um estudo realizado em um Hospital Universitário de Belo Horizonte, verificou também uma incidência significativa de afirmações da cabeceira elevada a $45^{\circ}$, como sendo o posicionamento adequado durante a oferta da dieta ${ }^{15}$.

Questionados quanto a capacidade de identificar se o paciente apresenta algum sinal ou sintoma da alteração da deglutição, quase que unanimidade confirmaram ser capaz de identificar a presença da disfagia, tendo apenas $2 \%$ técnicos de enfermagem e $8 \%$ enfermeiros que negaram ter esse conhecimento.

Temos que a maioria dos profissionais, sendo $92,3 \%$ dos enfermeiros e $88 \%$ dos técnicos de enfermagem, reconhecem que a tosse ou engasgo ao engolir, redução do estado de alerta e pneumonia aspirativa são sinais importantes na avaliação de uma alteração de deglutição.

Contudo, uma parcela $9 \%$ dos técnicos de enfermagem apenas consideram o sinal de tosse ou engasgo ao engolir como importante numa alteração de deglutição.

Como já citado anteriormente, a avaliação da disfagia deve ser realizada pelo fonoaudiólogo, profissional mais habilitado para avaliar e tratar as alterações de deglutição. Entretanto, conforme relaciona Werner*, a equipe de enfermagem também deve e pode contribuir na identificação, avaliação, controle, além de impedir as complicações relacionadas com a disfagia.

A maioria dos pacientes hospitalizados, de acordo com Schelp*, apresentam disfagia desde os primeiros dias de internação. Dessa forma, a equipe de enfermagem deve ajudar na prevenção de complicações, reduzindo o número de atos associados à disfagia através da observação dos sinais e sintomas presentes na alteração da deglutição, comunicando prontamente a equipe multiprofissional ${ }^{4}$.

Referente aos sinais e sintomas presentes na disfagia, 61,5\% dos enfermeiros confirmaram ser a disfonia, dispnéia, engasgo, tosse, queda de saturação durante a alimentação, assim como $72 \%$ dos técnicos de enfermagem.

Entretanto, uma proporção significativa de técnicos de enfermagem (23\%) afirmaram ser a presença de sialorréia, presença de restos alimentares em cavidade oral, recusa alimentar, demora para alimentar-se, xerostomia. Dessa forma, percebe-se uma possível incompreensão do conceito de xerostomia, que é o oposto de presença de sialorréia, tornando a alternativa incorreta apenas por essa oposição, já que há realmente presença de restos alimentares em cavidade oral, recusa alimentar, demora para alimentar-se e, presença da xerostomia, o que promove dificuldade na preparação, controle do bolo alimentar e na inicialização do processo da deglutição - fase oral, finalizam Costa et al. *

\footnotetext{
* Werner H. The benefits of the dysphagia clinical nurse specialist role. JNeurosci Nurs. 2005;37(4):212-5 apud ${ }^{15}$

* Schelp AO, Cola PC, Gatto AR, Silva RG, Carvalho LR. Incidência de disfagia orofaríngea após acidente vascular encefálico em hospital público de referência. Arq Neuropsiquiatr. 2004;62(2B):503-6 apud 15

* Costa HMC, Luz MOR, Carmona MJC, Cardoso E, Isisaki M, Júnior JOCA. Reintrodução da alimentação oral em pacientes traqueostomizados com terapia de nutrição enteral. Rev. bras. nutr. clín. 2003;18(4):168-172 apud ${ }^{16}$
} 
Consideravelmente, a maior parte dos pesquisados, $54 \%$ dos enfermeiros e $71 \%$ dos técnicos de enfermagem, confirmaram que ao perceber alguma alteração de deglutição no paciente internado, eles registram no prontuário, comunicam ao médico, comunicam o fonoaudiólogo, e no caso dos técnicos de enfermagem, comunicam ao enfermeiro, respeitando assim a hierarquia existente entre essa categoria profissional.

Mesmo que em pequena proporção, alguns profissionais da enfermagem ainda, demonstram acreditar no modelo de saúde em que o médico é o profissional exclusivo no tratamento dos doentes, onde o discurso médico prevalece sob qualquer outro conhecimento, onde não tem espaço para a colaboração da equipe multiprofissional.

Como já foi citado anteriormente, na assistência do paciente disfágico há o envolvimento de diversos profissionais, cada qual com a sua competência funcional e colaboração no restabelecimento da saúde desses pacientes, já que a disfagia remete a um diagnóstico e terapêutica complexos, afirma Logemann*.

Verifica-se que a grande maioria dos enfermeiros $(76,9 \%)$ consideram os seguintes diagnósticos como possibilidades para prescrição dos cuidados referentes a assistência ao paciente disfágico: padrão ineficaz de alimentação, risco de aspiração e dentição prejudicada, exceto o débito cardíaco diminuído.

No caso dos técnicos de enfermagem, a maior parte $(72 \%)$ consideram os mesmos diagnósticos de enfermagem que os enfermeiros para aassistênciaao paciente disfágico. Contudo, alguns (16\%)apenas consideraram como diagnóstico de enfermagem o padrão ineficaz de alimentação, deixando de lado, outros diagnósticos significativos na assistência ao paciente disfágico.

Os dados da pesquisa demonstram que todos os enfermeiros pesquisados (100\%) e $91 \%$ dos técnicos de enfermagem, ao admitir um paciente com fatores de risco para alguma alteração de deglutição, consideram como procedimentos essenciais, exceto: fazer nada, além da rotina normal de admissão do doente. Desse modo, confirmam que checar a via de alimentação e o tipo de dieta liberada, observar se o doente está consciente e alerta antes de oferecer via oral e observar se o doente deglute a saliva, são procedimentos fundamentais durante a admissão de novos pacientes que possam a vir apresentar alguma alteração da deglutição.

Sabe - se que a prática da enfermagem consiste na avaliação inicial do estado de saúde do paciente, identificando problemas atuais ou potenciais, assim também, como reconhecer a presença de alguma alteração de deglutição, diminuindo o número de mortes associadas à disfagia, já que a equipe de enfermagem passa maior parte do tempo junto ao paciente, finaliza Werner*.

Analisando os dados dos resultados, observa-se que $92,3 \%$ dos enfermeiros e $80 \%$ dos técnicos de enfermagem, não consideram como cuidado primordial, oferecer dieta por via alternativa de alimentação ou via oral para o paciente na postura deitado e com a cabeça fletida, pois como já foi citado anteriormente, a posição adequada para oferta da dieta por via oral, é sentado, desde que não haja impedimentos pelas condições clínicas que impossibilitem esse posicionamento.

Ainda, 7,7\% dos enfermeiros e $10 \%$ dos técnicos de enfermagem afirmam que não se deve oferecer dieta ou líquido ou medicação por via oral, se o paciente estiver sonolento ou com nível de consciência rebaixado.

Dessa forma, é possível sugerir que a equipe de enfermagem pesquisada reconhece satisfatoriamente quais os cuidados primordiais que devem ser realizados com o paciente disfágico, entre eles: realizar higiene oral adequada, observar o desempenho e aceitação da dieta por via oral, além de não oferecer dieta ou líquido ou medicação por via oral, se o paciente estiver sonolento ou com nível de consciência rebaixado.

\section{Considerações Finais}

A disfagia é uma condição clínica que requer a intervenção de uma equipe multidisciplinar, destacando o papel dos profissionais da enfermagem, que passam a maior parte do tempo junto ao paciente.

Diante dos resultados, verificou-se que a grande maioria dos dados remete que os profissionais do serviço de enfermagem do HRS apresentam um conhecimento satisfatório sobre a disfagia, seus fatores etiológicos, seus sinais e sintomas, consequências, e quais os cuidados pertinentes desenvolvidos na assistência ao paciente disfágico.

Apesar do reconhecimento dos pesquisados quanto a ser o fonoaudiólogo o profissional responsável pela reabilitação da disfagia, sabe-se que em muitas instituições hospitalares não contam com a atuação desse profissional, o que justificaria alguns índices inadequados ou incoerentes dos dados, onde muitos profissionais já trazem consigo uma

\footnotetext{
* Logeman JA. Oropharyngeal dysphagia and nutritional management. Current Opinion Clin Nutr Metabolic Care. 2007;10:611-4 apud ${ }^{7}$

* Werner H. The benefits of the dysphagia clinical nurse specialist role. JNeurosci Nurs. 2005;37(4):212-5 apud ${ }^{15}$
} 
bagagem de experiência superior ao tempo de existência dessa instituição, e não teriam tido até então, alguma interação com o serviço de Fonoaudiologia hospitalar.

Ainda, nota-se que nas instituições de ensino de enfermagem, os conteúdos programáticos relacionados às alterações de deglutição, e suas repercussões na condição de saúde do paciente internado, é de certa forma negligenciado, não abordando esse tema de maneira que contribua para que esses profissionais possam oferecer uma assistência segura e de qualidade ao paciente. Assim, evidencia-se a necessidade do aperfeiçoamento das disciplinas que abordem sobre a assistência ao paciente hospitalizado, ressaltando a importância e o papel da enfermagem na avaliação e reabilitação destes pacientes.

Durante a realização desse estudo, algumas limitações foram observadas quanto a abordagem aos sujeitos de pesquisa, visto a jornada de trabalho $12 \times 36$ horas, e assistência ao paciente, o que tornou impossível para alguns profissionais preencherem o questionário durante o plantão. Além disso, verifica-se a indisponibilidade de literatura atualizada em bibliotecas locais e regionais, bem como a inexistência do curso de Fonoaudiologia na região do sudoeste do Paraná.

Quanto às contribuições, temos o despertar dos profissionais na necessidade de visualizar o paciente como um todo, atentando para os aspectos relacionados a assistência integral; levantar de forma clara a carência de capacitação da equipe atuante na instituição sobre 0 assunto; além de aprimorar a formação dos profissionais da enfermagem sobre 0 distúrbio da deglutição e o cuidado integral do paciente hospitalizado.

Este estudo, de certa forma, estimula o desenvolvimento de novas pesquisas que abordem o tema transtorno de deglutição relacionado a assistência de enfermagem ao paciente disfágico, permitindo aos profissionais, não somente a prescrição dos diagnósticos, como também os cuidados indicados.

Por fim, sugere-se maiores investimentos na educação permanente direcionada ao cuidado integral do paciente disfágico, com orientações que visem o aprimoramento tanto teórico quanto prático da equipe de enfermagem do HRS, além da estimulação da intervenção multidisciplinar, favorecendo a recuperação global do paciente hospitalizado.

\section{Referências Bibliográficas}

1.Souza BBA, Martins C, Campos DJ, Balsini ID, Meyer LR. Nutrição e disfagia - guia para profissionais. Curitiba: Nutroclínica; 2003.

2.Prodomo LPV. Avaliação clínica fonoaudiológica das disfagias. In: Jotz GP, de Angelis EC, Barros APB. Tratado da deglutição e disfagia no adulto e na criança. Rio de Janeiro: Revinter; 2010.p.61-67.

3. Rangel FB. Disfagia no adulto: o papel do fonoaudiólogo e nutricionista [dissertação] [Internet]. São Paulo: Centro de Especialização em Fonoaudiologia Clínica (CEFAC); 1998 [acesso em 2014 set 4] Disponível: http://www.cefac.br/ library/teses/3c3ed55eb6effeecd18c80129e090034.pdf.

4. Ramiro D. Os enfermeiros são mais cobiçados do que os médicos. Você/ SA. [Internet] 2013 [acesso em 2014 set 12] Disponível: http://exame.abril.com.br/revista-voce-sa/edicoes/184/noticias/mais-cobicados-do-que-os-medicos.

5. Ministério da Saúde - Agência Nacional de Vigilância Sanitária. Resolução No 7/2010. Brasília: Ministério da Saúde - Agência Nacional de Vigilância Sanitária; 2010. Disponível: http://bvsms.saude.gov.br/bvs/saudelegis/anvisa/2010/ res0007_24_02_2010.html.

6. Cheregatti AL, Amorim CP. Enfermagem em unidade de terapia intensiva. São Paulo: Martinari; 2010.

7. Albini RMN, Soares VMN, Wolf AE, Gonçalves CGO. Conhecimento da enfermagem sobre cuidados a pacientes disfágicos internados em unidade de terapia intensiva. Rev. Cefac [Internet] 2013 [acesso em ago 2014 28];15(6):15121524. Disponivel: http://www.scielo.br/pdf/rcefac/2013nahead/45-12.pdf.

8. Faria HS, Barboza J, Pantano NP, Chagas LF. Instrumento prático para classificação de pacientes conforme Fugulin e Braden simultaneamente. Sobragen-

Encontro Científico - Competências, 2009. Moema: Anfiteatro do Hospital Alvorada; 2009. Disponível: http://www. sobragen.org.br/trabalhos_enenge/Trabalho\%20091.pdf

9.Vigna CP, Perroca MG. Utilização de sistema de classificação de pacientes e métodos de dimensionamento de pessoal de enfermagem. Arq Ciênc Saúde [Internet] 2007 [acesso em 2014 nov 3];14(1):8-12. Disponível: http://www. cienciasdasaude.famerp.br/racs_ol/vol-14-1/id215.pdf.

10. Conselho Federal de Enfermagem. Resolução No 293/2004. Rio de Janeiro: Conselho Federal de Enfermagem COFEN; 2004. Disponível: http://novo.portalcofen.gov.br/resoluo-cofen-2932004_4329.html. 
11. Fugulin FMT, Gaidzinski RR, Kurcgant P. Sistema de classificação de pacientes: identificação do perfil assistencial dos pacientes das unidades de internação do HU-USP. Rev Latino-am Enfermagem. 2005; 13(1):72-8.

12. Santos F, Rogenski NMB, Baptista CMC, Fugulin FMT. Sistema de classificação de pacientes: proposta de complementação do instrumento de Fugulin et al. Rev Latino-am Enfermagem [Internet] 2007 [acesso em ago 2014 28];15(5):980-985. Disponível: http://www.scielo.br/pdf/rlae/v15n5/pt_v15n5a14.pdf.

13. Padovani AR, Moraes DP, Mangili LD, Andrade CRF. Protocolo fonoaudiológico de avaliação do risco para disfagia (PARD). Rev. Soc. Bras. Fonoaudiologia [Internet]. 2007 [acesso em 2014 set 6];12(3):199-205. Disponível: http://www. scielo.br/pdf/rsbf/v12n3/a07v12n3.pdf.

14. Antunes MFC. Treinamento da equipe de enfermagem no cuidado do doente com disfagia orofaríngea na UTI: uma proposta de educação continuada [dissertação] [Internet]. São Paulo: Faculdade de Ciências Médicas da Santa Casa de São Paulo; 2010. [acesso em 2014 set 4] Disponível: http://www.dominiopublico.gov.br/download/texto/cp126596.pdf. 15. Guedes LU, Vicente LCC, Paula CM, Oliveira E, Andrade EA, Barcelos WCO. Conhecimento dos profissionais da enfermagem que assistem pacientes com alterações da deglutição em um hospital universitário de Belo Horizonte. Rev. Soc. Bras Fonoaudiologia [Internet] 2009 [acesso em 2014 set 4];14(3);372-80. Disponível: http://www.scielo.br/pdf/rsbf /v14n3/v14n3a14.pdf.

16. Oliveira MMG, Teruel SL, Lima JL, Bergamasco CM, Aquino RC. Terapia nutricional em disfagia: a importância do acompanhamento nutricional. RBCS [Internet] 2008 [acesso em 2014 set 3];VI(16). Disponível: http://seer.uscs.edu.br/ index.php/revista_ciencias_saude/article/view/382.

\section{Ana Karenina Cioatto}

Endereço para correspondência - Rua: Pernambuco, n 1360 - Ap 02, Bairro: Industrial, CEP: 85601-300, Cidade: Francisco Beltrão - PR, Brasil

E-mail: karenina.batista@gmail.com

Lattes: http://lattes.cnpq.br/2052088485827777

Nádia Aparecida Zanella - nanizanella@hotmail.com

Enviado em 20 de novembro de 2013. Aceito em 30 de março de 2015. 
